\title{
Molecular Bases of Cutaneous and Uveal Melanomas
}

\author{
Sudeep Gaudi ${ }^{1}$ and Jane L. Messina ${ }^{2}$ \\ ${ }^{1}$ Department of Pathology and Cell Biology, University of South Florida College of Medicine, 12901 Bruce B. Downs Boulevard, \\ MDC 11, Tampa, FL 33612, USA \\ ${ }^{2}$ Department of Pathology, H. Lee Moffitt Cancer Center, 2nd Floor, 12902 Magnolia Dr., Tampa, FL 33612, USA
}

Correspondence should be addressed to Sudeep Gaudi, sgaudi@health.usf.edu

Received 15 March 2011; Revised 27 May 2011; Accepted 30 May 2011

Academic Editor: Gerardo Ferrara

Copyright ( $\odot 2011$ S. Gaudi and J. L. Messina. This is an open access article distributed under the Creative Commons Attribution License, which permits unrestricted use, distribution, and reproduction in any medium, provided the original work is properly cited.

\begin{abstract}
Intensive research in recent years has begun to unlock the mysteries surrounding the molecular pathogenesis of melanoma, the deadliest of skin cancers. The high-penetrance, low-frequency susceptibility gene CDKN2A produces tumor suppressor proteins that function in concert with p53 and retinoblastoma protein to thwart melanomagenesis. Aberrant CDKN2A gene products have been implicated in a great many cases of familial cutaneous melanoma. Sporadic cases, on the other hand, often involve constitutive signal transduction along the mitogen-activated protein kinase (MAPK) pathway, with particular focus falling upon mutated RAS and RAF protooncogenes. The proliferative effects of the MAPK pathway may be complemented by the antiapoptotic signals of the PI3K/AKT pathway. After skin, melanoma most commonly affects the eye. Data for the constitutive activation of the MAPK pathway in uveal melanoma exists as well, however, not through mutations of RAS and RAF. Rather, evidence implicates the proto-oncogene GNAQ. In the following discussion, we review the major molecular pathways implicated in both familial and sporadic cutaneous melanomagenesis, the former accounting for approximately $10 \%$ of cases. Additionally, we discuss the molecular pathways for which preliminary evidence suggests a role in uveal melanomagenesis.
\end{abstract}

\section{Introduction}

Melanoma remains a disproportionate cause of death among skin cancers $[1,2]$. Currently, early diagnosis followed by complete surgical removal of the tumor offers the best hope for cure [3]. Once advanced, melanoma is notoriously resistant to medical interventions [3]. Thus, great interest lies in the discovery of new therapeutic options that may improve the prognoses of those afflicted with this unforgiving disease.

New insights into the development and/or progression of cutaneous melanoma have been achieved through the study of its molecular pathogenesis. Key molecules at crucial junctions have been identified and have begun serving as potential targets for clinicians tasked with containing this lethal disease.

After skin, primary melanoma most commonly affects the eye [4]. The two most commonly employed modalities for the treatment of uveal melanoma, the most lethal of ocular melanomas, are radiation therapy and enucleation [5]. Despite these valiant efforts at local disease control, up to $50 \%$ of patients succumb to their disease, and impact on patient survival remains questionable at best [6]. Thus, a great need for improved therapy exists for the treatment of uveal melanoma.

In the following discussion, we review the major molecular pathways implicated in both familial and sporadic cutaneous melanomagenesis, the former accounting for approximately $10 \%$ of cases [7]. Additionally, we discuss the molecular pathways for which preliminary evidence suggests a role in uveal melanomagenesis.

\section{Familial Cutaneous Melanoma}

Knowledge of some of the earliest molecular pathways involved in melanomagenesis derived from investigations of familial cutaneous melanoma. In affected individuals, a complex network of interrelated pathways functions to promote cellular proliferation and cellular survival.

2.1. CDKN2A. The best-characterized high-penetrance susceptibility gene predisposing to cutaneous melanoma is CDKN2A [3, 8-12]. This gene is located on chromosome 
9p21 and encodes two distinct tumor-suppressor proteinsp14/ARF and p16/INK4a-implicated in the pathogenesis of $25-40 \%$ of familial cutaneous melanomas (Figure 1) [3, 13]. The former deters melanomagenesis through its indirect effect on p53, a tumor-suppressor protein also known as "the guardian of the genome." Upon sensing DNA damage, p53 promotes the transcription of numerous genes involved in cell cycle arrest and/or apoptosis. Simply stated, if DNA damage can be repaired during cell cycle arrest, the cell returns to its normal functional state. If damage is irreparable, however, p53 stimulates the transcription of microRNAs (miRNAs), specifically the mir34 family of miRNAs, which silence the translation of proproliferative and antiapoptotic transcripts resulting in either quiescence/senescence or apoptosis, respectively.

Under homeostatic conditions, p53 maintains a relatively short half-life due to the function of human homolog of murine Mdm2 (HDM2), a protein that ubiquitinates other proteins for destruction. When the cell is stressed, however, p14/ARF binds to and inhibits the function of HDM2 allowing p53 to escape ubiquitination. Mutated p14/ARF, on the other hand, is unable to bind and suppress HDM2, allowing it to mark p53 for destruction. With less p53 available to identify damaged DNA, genomic instability results, predisposing the afflicted individual to the development of cutaneous melanoma.

p16/INK4a functions in concert with retinoblastoma protein $(\mathrm{RBp})$, another tumor-suppressor protein, to regulate the Gap $1\left(\mathrm{G}_{1}\right)$ phase of the cell cycle. During this phase, cells can exit the cell cycle into quiescence or senescence, or make the necessary preparations to progress onward into the $S$ phase of the cycle. Specifically, phosphorylation of $\mathrm{RBp}$, which is partly dependent upon the cyclin $\mathrm{D}$ CDK4/6 complex, is necessary for the transcription of genes encoding cyclin E, a protein that is required for the initiation of DNA replication in the $S$ phase. p16/INK4a inhibits the phosphorylation of RBp by inactivating the cyclin D-CDK4/6 complex and consequently prevents the cell progression through the cell cycle. When p16 expression is compromised, and so is RBp's regulatory control on the cell cycle.

2.2. CDK4. Linkage studies have allowed the identification of another high-penetrance, low-frequency melanoma susceptibility gene, CDK4, which is mutated in three cutaneous melanoma kindreds worldwide [3, 9-11]. Located on chromosome 12q14, CDK4 encodes cyclin-dependent kinase 4 protein, a constituent of the CDK4/6 complex discussed above (Figure 1). Germline mutations that activate this gene occur at codon 24 (Arg24Cys and Arg24His) and render the CDK4/ 6 complex resistant to p16 inhibition. Similar to the case of aberrations in the CDKN2A gene, mutations in CDK4 lend to an increased risk for cutaneous melanomagenesis.

\section{Sporadic Cutaneous Melanoma}

3.1. RAS/RAF/MEK/ERK Signaling Pathway. Constitutive stimulation of the mitogen-activated protein kinase (MAPK) pathway, which regulates cellular proliferation, has been implicated in up to $90 \%$ of cutaneous melanomas $[3,9,11$, $12,14,15]$. The MAPK pathway exerts its effects through signal transduction along the cascade of RAS, RAF, MAPK extracellular signal-regulated kinase (MEK), and extracellular signal-regulated kinase (ERK) (Figure 2). MAPK signaling initiates when a receptor tyrosine kinase in the cell membrane binds its respective ligand-an event that results in the activation of RAS, a membrane-bound protein with GTPase activity. Activated RAS then recruits RAF, a cytosolic serine-threonine-specific protein kinase, to the plasma membrane. Through phosphorylation, RAS activates RAF, which in turn phosphorylates and activates MEK. Activated MEK activates ERK, which induces several proliferative and survival processes, one of which is activation of the cyclin D-CDK4/6 complex (discussed above) upon translocation to the nucleus.

3.1.1. $c$-KIT. c-KIT, a protooncogene that encodes the type III receptor tyrosine kinase KIT, was first identified in 1987 as a result of sequence similarity to the Hardy-Zuckerman 4 feline sarcoma virus oncogene, v-KIT [16]. Upon binding its ligand, stem cell factor (SCF), KIT undergoes receptor dimerization, autophosphorylation, and activation of its intracellular tyrosine kinase domain $[8,17]$. Once activated, KIT is capable of stimulating downstream signaling pathways, such as MAPK $[8,17,18]$.

Activating mutations and/or gene amplification of KIT are now being described in significant subsets of melanomas [19-23]. One study, in particular, recognized such aberrations in $39 \%$ of mucosal melanomas, $36 \%$ of acral melanomas, and 28\% of melanomas arising in chronically sun-damaged skin (as defined by the presence of solar elastosis on review of histopathology) — anatomic sites at which $B R A F$ mutations occur far less frequently [22].

The most prevalent KIT mutations are L576P (exon 11), K642E (exon 13), V559A (exon 11), and D816H (exon 17) $[17,24]$. These mutations are thought to promote the constitutive activation of KIT either through precluding the protein from assuming its default autoinhibited conformation, or by promoting its dimerization in the absence of SCF $[25,26]$. The precise manner in which constitutive KIT activation promotes melanomagenesis remains unclear.

3.1.2. NRAS. First implicated in sarcoma in rats, HRAS and KRAS - members of the RAS family of protooncogeneswere recognized for their ability to undergo activating transformation under the influence of the Harvey and Kirsten sarcoma viruses. A third member of the RAS family, NRAS, was subsequently identified in human neuroblastoma cells. Of these three protooncogenes, activating mutations in NRAS occur most frequently in melanocytes and have been identified in nearly one-third of all melanomas $[3,12$, 15]. The most commonly documented NRAS aberration in melanoma is a missense mutation at codon 61 (Q61R), which results in the substitution of arginine in place of glutamine and impairs GTP hydrolysis locking the protein in a state of constitutive activation [27]. In comparison to other solid tumors, mutations in RAS do not occur with as high as a frequency in melanoma [3]. Nonetheless, in the absence of 


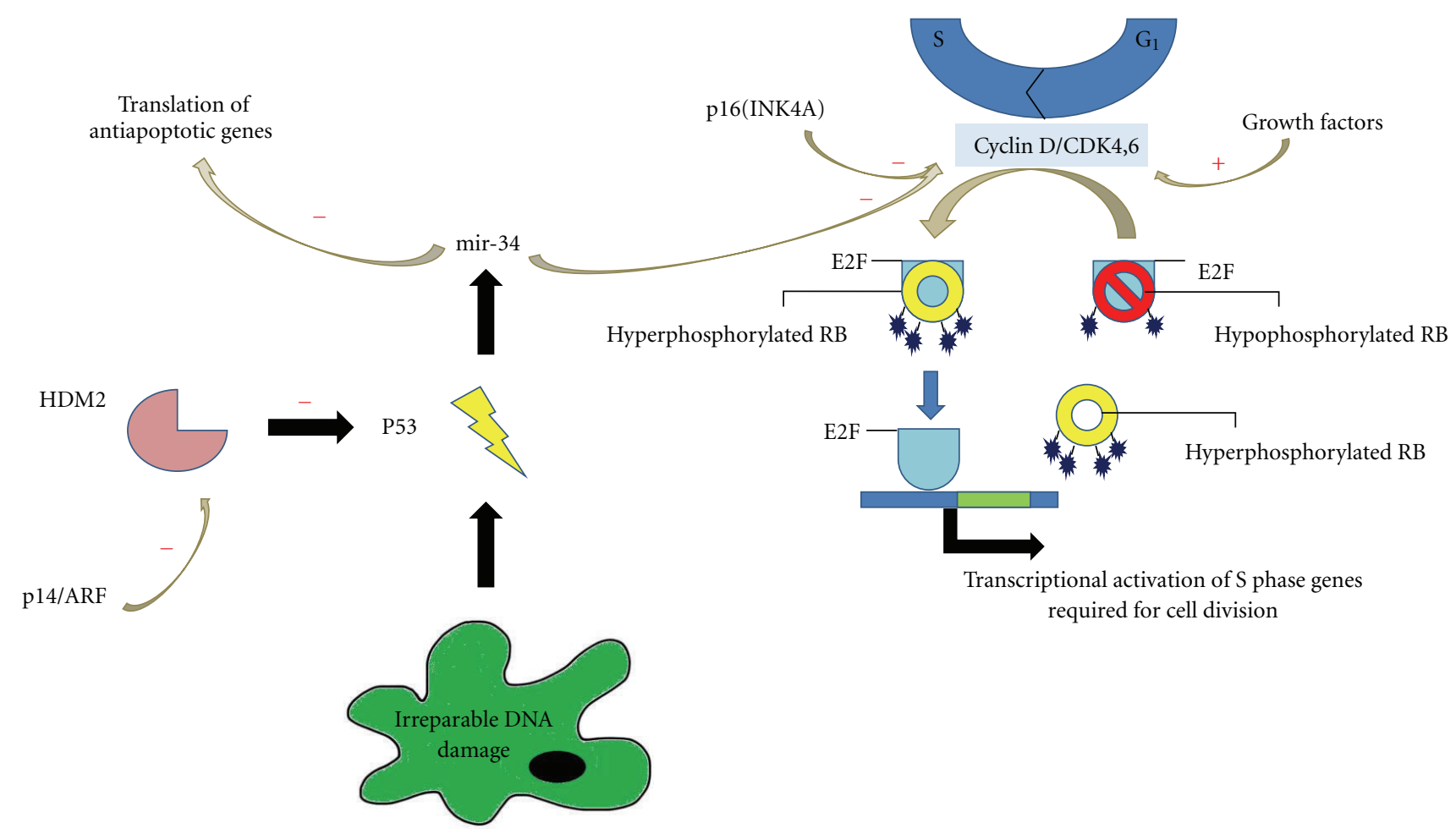

FIGURE 1: Roles of p14/ARF, p16(INK4A), and cyclin-dependent kinase 4 protein in cellular proliferation and survival. Loss of function of any of these molecules has been implicated in the pathogenesis of Familial Cutaneous Melanoma.

the inhibitory effects of p16/INK4a, oncogenic RAS has been shown to induce melanoma and appears to have an integral function in tumor maintenance and progression $[28,29]$.

3.1.3. BRAF. Each of the three RAF protooncogenes-ARAF, $B R A F$, and $C R A F$ - have been identified in mammals. While $A R A F$ and $C R A F$ mutations are rare in human cancers, a significant percentage of human malignancies have been shown to harbor activating mutations in $B R A F$, with the highest rate occurring in melanoma $[3,9,11,12]$. BRAF mutations in melanoma tend to occur at anatomic sites exposed to intermittent, rather than chronic, sun damage. With approximately $70 \%$ of cases harboring such a mutation, $B R A F$ is the most commonly mutated protooncogene in cutaneous melanoma. Furthermore, a significant proportion of both benign and dysplastic melanocytic nevi have been shown to harbor mutation of $B R A F$ as well, suggesting a relatively early event in melanomagenesis $[3,11,12,30]$.

Greater than $90 \%$ of BRAF mutations in melanoma result from a single base missense mutation $(\mathrm{T} \rightarrow \mathrm{A})$ at codon 1799 that leads to the substitution of valine in favor of glutamic acid at position 600 of the BRAF protein [31]. This alteration introduces a conformational change in BRAF's kinase domain, which can lead to a 480 -fold increase in kinase activity when compared to that of wild-type BRAF [32]. However, the story is not as simple as this may suggest. While mutated BRAF induces uncontrolled proliferation in melanoma, it lends to senescence in benign melanocytic nevi. Furthermore, a subset of melanocytes is able to bypass the senescent response and undergo malignant transformation upon the accumulation of additional insults.
3.2. PI3K/AKT Signaling Pathway. Activated RAS also triggers the phosphatidylinositol 3-kinase (PI3K)/AKT pathway, which conducts antiapoptotic signals that complement the proliferative effects of the MAPK pathway (Figure 2) [3, $11,12,15]$. PI3K phosphorylates phosphatidylinositols of the cell membrane to produce phosphatidylinositol-3,4,5triphosphate (PIP3). PIP3 then recruits the serine/threonine kinase AKT, also known as protein kinase $B$, to the cell membrane. Activation of AKT requires phosphorylation by PDK1,2. AKT then promotes cell survival by phosphorylating several substrates, such as BAD (a member of the bcl-2 family of apoptosis regulator proteins) and HDM2 (discussed above). AKT also inactivates the complex formed by TSC1 and TSC2, tumor-suppressor proteins known to be mutated in tuberous sclerosis. Inactivation of the TSC1/ TSC2 complex stimulates activity of mammalian target of rapamycin (mTOR), a kinase that promotes the uptake of nutrients necessary for cellular growth.

3.2.1. PTEN. The phosphatase and tensin homolog (PTEN) gene is located on chromosome 10 and encodes a tumorsuppressor protein that functions as both a lipid and protein phosphatase $[3,11,12,15]$. Specifically, the PTEN protein degrades the products of PI3K, thereby antagonizing the activity of the PI3K/AKT pathway. This results in increased apoptosis and decreased tumorigenesis. Loss of functional PTEN protein has been demonstrated in approximately $20 \%$ of primary cutaneous melanomas and has been shown to positively correlate with increasing Breslow thickness [33]. Frequency of mutations and deletions only partly account for PTEN loss in melanoma samples, suggesting epigenetic 


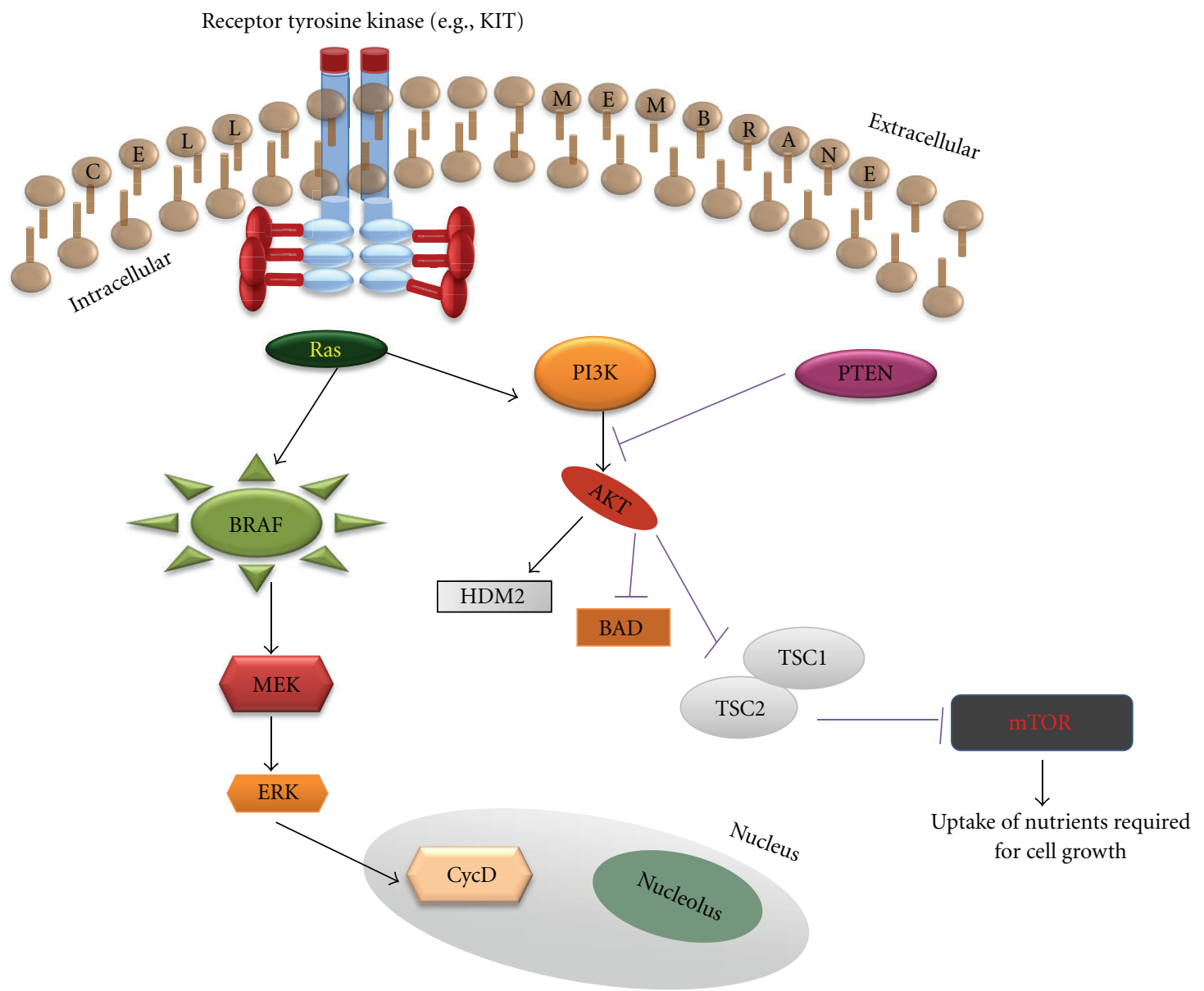

FIGURE 2: MAPK and PI3K/AKT pathways regulate cellular proliferation and survival. Aberrant functioning of these pathways has been implicated in the pathogenesis of sporadic cutaneous melanoma.

mechanisms [34]. PTEN losses occur with greater frequency in primary melanomas when compared with benign melanocytic nevi, but with lesser frequency when compared with metastatic melanomas [34, 35]. Thus, PTEN losses appear to occur in later stages of melanomagenesis and may contribute to the transformation of a benign melanocytic proliferation into an invasive melanoma.

\section{Ocular Melanoma}

Melanomas of the eye are categorized as either conjunctival or uveal, with the latter being further divided between anterior uveal melanomas (iris) and posterior uveal melanomas (ciliary and choroidal). Not surprisingly, differences in the histopathology and/or clinical presentation of these neoplasms exist, and the most striking distinction is in prognosis $[36,37]$. Melanomas of the conjunctiva and iris have relatively good outcomes, while survival rates of those with posterior uveal melanoma markedly decline. Specifically, the 5-year mortality rate due to metastasis of ciliary body or choroidal melanomas is approximately $30 \%$, compared to about 3\% for iris melanomas [37]. Thus, a greater urgency in exploring the molecular mechanisms of posterior uveal melanomagenesis in the hopes of developing new and effective therapies is understandable.

\section{Uveal Melanoma}

The identification of susceptibility genes through linkage analysis has not proved fruitful due to the low occurrence of uveal melanoma in the familial setting. Furthermore, while CDKN2A is implicated in $25-40 \%$ of familial cutaneous melanomas, the germline mutation infrequently occurs in the rare uveal melanoma-prone families studied [38]. Finally, data implicating the protooncogenes NRAS and BRAF is not as convincing in uveal melanomagenesis as it is in sporadic cutaneous melanomagenesis [39-41]. Nonetheless, evidence for the constitutive activation of the MAPK pathway in uveal melanoma exists, suggesting involvement of a disparate protooncogene [41].

5.1. GNAQ. Screening of potential oncogenes that may activate the MAPK pathway has led to the discovery of mutations in GNAQ, a stimulatory $\alpha_{\mathrm{q}}$ subunit of heterotrimeric G proteins $(\mathrm{G} \alpha \beta \gamma)$ [42-44]. Upon the binding of an extracellular signal molecule to a G-protein-coupled receptor on the cell surface, GDP bound to the $\mathrm{G} \alpha$ subunit of $\mathrm{G} \alpha \beta \gamma$ is replaced with GTP, allowing for the dissociation of $\mathrm{G} \alpha$ from $\mathrm{G} \beta \gamma$. No longer tethered to $\mathrm{G} \beta \gamma, \mathrm{G} \alpha_{q}$ stimulates phospholipase $\mathrm{C} \beta$, until its intrinsic GTPase activity renders it unable to do 


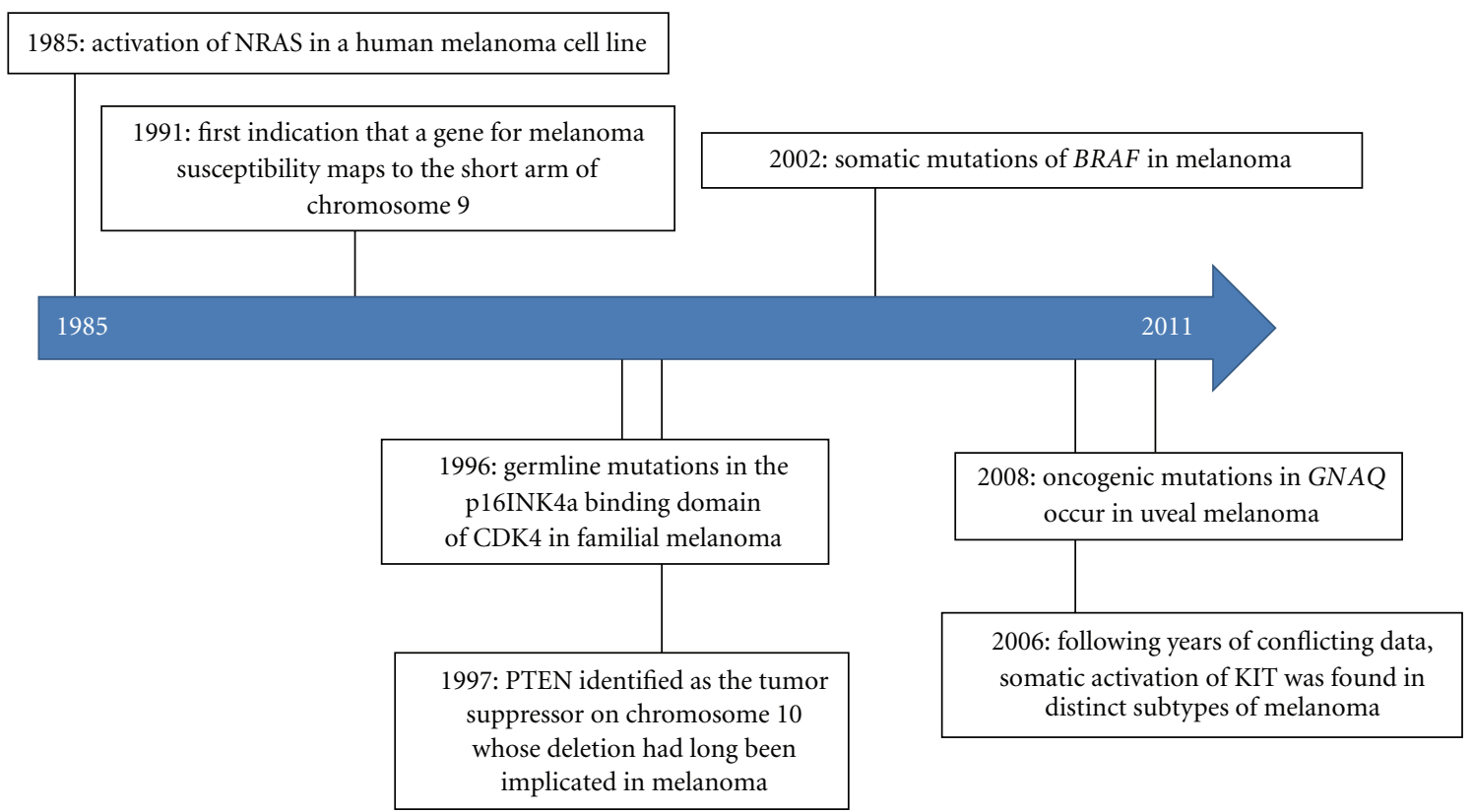

FIGURE 3: Timeline highlighting pivotal discoveries of key molecular players discussed in this paper on melanomagenesis.

so. Phospholipase $\mathrm{C} \beta$ hydrolyzes phosphatidylinositol 4,5bisphosphonate (PIP2) into two second messengers: inositol 1,4,5-triphosphate (IP3) and diacylglycerol (DAG). IP3 functions to increase cytosol calcium levels by releasing the cation from endoplasmic reticulum stores. Calcium then activates conventional isoforms of the serine/threonine kinase, protein kinase $\mathrm{C}$, while DAG activates both conventional and novel isoforms of the kinase. Finally, protein kinase $\mathrm{C}$ stimulates the RAF/MEK/ERK pathway, which induces several proliferative and survival processes, as discussed above.

Mutation of GNAQ at codon 209 prevents the hydrolysis of GTP and locks GNAQ in its active, GTP-bound state. Constitutive activation of GNAQ is tantamount to oncogenic activation of the MAPK cascade and accounts for approximately $50 \%$ of uveal melanomas [43]. While inhibitors of GNAQ, phospholipase $\mathrm{C} \beta$, or protein kinase $\mathrm{C}$ isoforms have yet to be developed, small, preliminary studies involving the downstream inhibition of MEK have encouraged investigation through formal clinical trials [45]. The results of these larger studies may offer much needed hope to those afflicted with this aggressive disease.

\section{Conclusion}

Many of the pivotal discoveries regarding the key molecular players discussed in this paper are highlighted on the timeline pictured in Figure 3 [22, 42, 46-50]. While much progress has been made in unlocking the mysteries surrounding the molecular pathogenesis of cutaneous melanoma, the story is far from complete. Just as researchers are beginning to understand the mechanisms by which activating mutations in the RAS and RAF protooncogenes lead to proliferative and antiapoptotic effects, evidence is mounting for the role of constitutive MAPK activity in tumor evasion of immune surveillance, suppression of immune response, tumor angiogenesis, and metastatic dissemination [51-62]. Furthermore, approximately $40 \%$ of melanoma kindreds harbor CDKN2A mutations, and significantly less perpetuate CDK4 mutations, thus the genetic basis of a substantial proportion of cases of familial cutaneous melanoma clearly remains unresolved [7, 63]. Genomewide association studies have enabled the identification of several low-penetrance, high-frequency susceptibility genes $[64,65]$. Analyses of these genes will likely reveal additional molecular pathways involved in melanoma formation and/or progression.

While the battle against uveal melanoma may not be as far along as that against its cutaneous counterpart, lines have been drawn in the sand, and war is under way. In addition to taking aim at the constitutive activation of the MAPK pathway, researchers have discovered potential targets in the $\mathrm{PI}$ /AKT and insulin growth factor (IGF) signaling pathways, mTOR, protooncogene c-MET, and tumor-suppressor breast cancer-1 (BRCA1)-associated protein-1 (BAP1), early onset [43]. While clinical trials are under way to determine if aberrations in the aforementioned molecules and pathways can be manipulated to stifle and/or reverse uveal melanomagenesis, the need for intervention at more than just one critical junction will likely be needed.

Despite the plethora of questions that remain, the potential of this early research to benefit disease-stricken patients is already being realized. Preliminary studies employing selective inhibitors against key players detailed in the aforementioned discussion have demonstrated encouraging results $[43,66,67]$. Whether these particular treatments prove effective in rigorous clinical trials remains to be seen. Regardless, researchers are reassured in seeking a molecular basis for the treatment of melanoma. 


\section{Acknowledgments}

The authors would like to thank Keiran Smalley, Ph.D. and Raymond M. Fertig, B. S., M. B. A. for their kind contributions of the graphics used in this paper.

\section{References}

[1] National Cancer Institute SEER Stat Fact Sheets, "Melanoma of the skin," 2010, http://seer.cancer.gov/statfacts/html/melan. html.

[2] National Cancer Institute Skin Cancer, 2010, http://www .cancer.gov/cancertopics/types/skin.

[3] N. Ibrahim and F. G. Haluska, "Molecular pathogenesis of cutaneous melanocytic neoplasms," Annual Review of Pathology, vol. 4, pp. 551-579, 2009.

[4] A. E. Chang, L. H. Karnell, and H. R. Menck, "The national cancer data base report on cutaneous and noncutaneous melanoma: a summary of 84,836 cases from the past decade: the American College of Surgeons Commission on Cancer and the American Cancer Society," Cancer, vol. 83, no. 8, pp. 16641678, 1998.

[5] N. V. Laver, M. E. McLaughlin, and J. S. Duker, "Ocular melanoma," Archives of Pathology and Laboratory Medicine, vol. 134, no. 12, pp. 1778-1784, 2010.

[6] B. Damato, "Does ocular treatment of uveal melanoma influence survival," British Journal of Cancer, vol. 103, no. 3, pp. 285-290, 2010.

[7] I. Yeh and B. C. Bastian, "Genome-wide associations studies for melanoma and nevi," Pigment Cell and Melanoma Research, vol. 22, no. 5, pp. 527-528, 2009.

[8] K. B. Calder and M. B. Morgan, "Carcinogenic pathway of malignant melanoma," in Mechanisms of Oncogenesis: An Update on Tumorigenesis , D. Coppola, Ed., pp. 149-157, Springer, 2010.

[9] K. D. Meyle and P. Guldberg, "Genetic risk factors for melanoma," Human Genetics, vol. 126, no. 4, pp. 499-510, 2009.

[10] A. A. Nelson and H. Tsao, "Melanoma and genetics," Clinics in Dermatology, vol. 27, no. 1, pp. 46-52, 2009.

[11] G. Palmieri, M. Capone, M. L. Ascierto et al., "Main roads to melanoma," Journal of Translational Medicine, vol. 7, Article ID 86, 2009.

[12] A. Sekulic, P. Haluska, A. J. Miller et al., "Malignant melanoma in the 21st century: the emerging molecular landscape," Mayo Clinic Proceedings, vol. 83, no. 7, pp. 825-846, 2008.

[13] L. Chin, "The genetics of malignant melanoma: lessons from mouse and man," Nature Reviews Cancer, vol. 3, no. 8, pp. 559-570, 2003.

[14] K. S. Smalley, "Understanding melanoma signaling networks as the basis for molecular targeted therapy," Journal of Investigative Dermatology, vol. 130, no. 1, pp. 28-37, 2010.

[15] Y. Kong, S. M. Kumar, and X. Xu, "Molecular pathogenesis of sporadic melanoma and melanoma-initiating cells," Archives of Pathology and Laboratory Medicine, vol. 134, no. 12, pp. 1740-1749, 2010.

[16] Y. Yarden, W. J. Kuang, T. Yang-Feng et al., "Human protooncogene c-kit: a new cell surface receptor tyrosine kinase for an unidentified ligand," EMBO Journal, vol. 6, no. 11, pp. 3341-3351, 1987.
[17] S. E. Woodman and M. A. Davies, "Targeting KIT in melanoma: a paradigm of molecular medicine and targeted therapeutics," Biochemical Pharmacology, vol. 80, no. 5, pp. 568-574, 2010.

[18] K. T. Flaherty, F. S. Hodi, and B. C. Bastian, "Mutationdriven drug development in melanoma," Current Opinion in Oncology, vol. 22, no. 3, pp. 178-183, 2010.

[19] C. R. Antonescu, K. J. Busam, T. D. Francone et al., "L576P KIT mutation in anal melanomas correlates with KIT protein expression and is sensitive to specific kinase inhibition," International Journal of Cancer, vol. 121, no. 2, pp. 257-264, 2007.

[20] A. Ashida, M. Takata, H. Murata, K. Kido, and T. Saida, "Pathological activation of KIT In metastatic tumors of acral and mucosal melanomas," International Journal of Cancer, vol. 124, no. 4, pp. 863-868, 2009.

[21] C. Beadling, E. Jacobson-Dunlop, F. S. Hodi et al., "KIT gene mutations and copy number in melanoma subtypes," Clinical Cancer Research, vol. 14, no. 21, pp. 6821-6828, 2008.

[22] J. A. Curtin, K. Busam, D. Pinkel, and B. C. Bastian, "Somatic activation of KIT in distinct subtypes of melanoma," Journal of Clinical Oncology, vol. 24, no. 26, pp. 4340-4346, 2006.

[23] R. S. Rivera, H. Nagatsuka, M. Gunduz et al., "C-kit protein expression correlated with activating mutations in KIT gene in oral mucosal melanoma," Virchows Archiv, vol. 452, no. 1, pp. 27-32, 2008.

[24] K. S. Smalley, V. K. Sondak, and J. S. Weber, "C-KIT signaling as the driving oncogenic event in sub-groups of melanomas," Histology and Histopathology, vol. 24, no. 5, pp. 643-650, 2009.

[25] J. Lennartsson, P. Blume-Jensen, M. Hermanson, E. Pontén, M. Carlberg, and L. Rönnstrand, "Phosphorylation of Shc by Src family kinases is necessary for stem cell factor receptor/ckit mediated activation of the Ras/MAP kinase pathway and cfos induction," Oncogene, vol. 18, no. 40, pp. 5546-5553, 1999.

[26] C. D. Mol, D. R. Dougan, T. R. Schneider et al., "Structural basis for the autoinhibition and STI-571 inhibition of c-Kit tyrosine kinase," Journal of Biological Chemistry, vol. 279, no. 30, pp. 31655-31663, 2004.

[27] A. Platz, S. Egyhazi, U. Ringborg, and J. Hansson, "Human cutaneous melanoma; a review of NRAS and BRAF mutation frequencies in relation to histogenetic subclass and body site," Molecular Oncology, vol. 1, no. 4, pp. 395-405, 2008.

[28] J. Ackermann, M. Frutschi, K. Kaloulis, T. McKee, A. Trumpp, and F. Beermann, "Metastasizing melanoma formation caused by expression of activated N-Ras ${ }^{\mathrm{Q} 61 \mathrm{~K}}$ on an INK4a-deficient background," Cancer Research, vol. 65, no. 10, pp. 4005-4011, 2005.

[29] L. Chin, A. Tam, J. Pomerantz et al., "Essential role for oncogenic ras in tumour maintenance," Nature, vol. 400, no. 6743, pp. 468-472, 1999.

[30] P. M. Pollock, U. L. Harper, K. S. Hansen et al., "High frequency of BRAF mutations in nevi," Nature Genetics, vol. 33, no. 1, pp. 19-20, 2003.

[31] I. Puzanov and K. T. Flaherty, "Targeted molecular therapy in melanoma," Seminars in Cutaneous Medicine and Surgery, vol. 29, no. 3, pp. 196-201, 2010.

[32] P. T. C. Wan, M. J. Garnett, S. M. Roe et al., "Mechanism of activation of the RAF-ERK signaling pathway by oncogenic mutations of B-RAF," Cell, vol. 116, no. 6, pp. 855-867, 2004.

[33] V. K. Goel, A. J. F. Lazar, C. L. Warneke, M. S. Redston, and F. G. Haluska, "Examination of mutations in BRAF, NRAS, and PTEN in primary cutaneous melanoma," Journal of Investigative Dermatology, vol. 126, no. 1, pp. 154-160, 2006. 
[34] A. Mirmohammadsadegh, A. Marini, S. Nambiar et al., "Epigenetic silencing of the PTEN gene in melanoma," Cancer Research, vol. 66, no. 13, pp. 6546-6552, 2006.

[35] H. Tsao, V. K. Goel, H. Wu, G. Yang, and F. G. Haluska, "Genetic interaction between NRAS and BRAF mutations and PTEN/MMAC1 inactivation in melanoma," Journal of Investigative Dermatology, vol. 122, no. 2, pp. 337-341, 2004.

[36] A. Zembowicz, R. V. Mandal, and P. Choopong, "Melanocytic lesions of the conjunctiva," Archives of Pathology and Laboratory Medicine, vol. 134, no. 12, pp. 1785-1792, 2010.

[37] "Intraocular (Eye) melanoma treatment," 2007, http://www .cancer.gov/cancertopics/pdq/treatment/intraocularmelano$\mathrm{ma} /$ HealthProfessional.

[38] A. M. Goldstein, S. N. Stacey, J. H. Olafsson et al., "CDKN2A mutations and melanoma risk in the Icelandic population," Journal of Medical Genetics, vol. 45, no. 5, pp. 284-289, 2008.

[39] F. Cruz, B. P. Rubin, D. Wilson et al., "Absence of BRAF and NRAS mutations in uveal melanoma," Cancer Research, vol. 63, no. 18, pp. 5761-5766, 2003.

[40] D. Rimoldi, S. Salvi, D. Liénard et al., "Lack of BRAF mutations in uveal melanoma," Cancer Research, vol. 63, no. 18, pp. 5712-5715, 2003.

[41] W. Zuidervaart, F. Van Nieuwpoort, M. Stark et al., "Activation of the MAPK pathway is a common event in uveal melanomas although it rarely occurs through mutation of BRAF or RAS," British Journal of Cancer, vol. 92, no. 11, pp. 2032-2038, 2005.

[42] M. D. Onken, L. A. Worley, M. D. Long et al., "Oncogenic mutations in GNAQ occur early in uveal melanoma," Investigative Ophthalmology and Visual Science, vol. 49, no. 12, pp. 5230-5234, 2008.

[43] M. Patel, E. Smyth, P. B. Chapman et al., "Therapeutic implications of the emerging molecular biology of uveal melanoma," Clinical Cancer Research, vol. 17, no. 8, pp. 20872100, 2011.

[44] C. D. Van Raamsdonk, V. Bezrookove, G. Green et al., "Frequent somatic mutations of GNAQ in uveal melanoma and blue naevi," Nature, vol. 457, no. 7229, pp. 599-602, 2009.

[45] "Phase II trial of temozolomide versus AZD6244 in patients with metastatic uveal melanoma," 2011, http://www.mskcc. $\mathrm{org} / \mathrm{mskcc} / \mathrm{html} / 2270 . \mathrm{cfm}$ ?IRBNO =10-053.

[46] H. Davies, G. R. Bignell, C. Cox et al., "Mutations of the BRAF gene in human cancer," Nature, vol. 417, no. 6892, pp. 949954, 2002.

[47] D. J. Nancarrow, G. J. Mann, E. A. Holland et al., "Confirmation of chromosome 9p linkage in familial melanoma," American Journal of Human Genetics, vol. 53, no. 4, pp. 936-942, 1993.

[48] R. A. Padua, N. C. Barrass, and G. A. Currie, "Activation of N-ras in a human melanoma cell line," Molecular and Cellular Biology, vol. 5, no. 3, pp. 582-585, 1985.

[49] P. A. Steck, M. A. Pershouse, S. A. Jasser et al., "Identification of a candidate tumour suppressor gene, MMAC1, at chromosome 10q23.3 that is mutated in multiple advanced cancers," Nature Genetics, vol. 15, no. 4, pp. 356-362, 1997.

[50] L. Zuo, J. Weger, Q. Yang et al., "Germline mutations in the p16(INK4a) binding domain of CDK4 in familial melanoma," Nature Genetics, vol. 12, no. 1, pp. 97-99, 1996.

[51] U. Benbow, G. B. Tower, C. A. Wyatt, G. Buttice, and C. E. Brinckerhoff, "High levels of MMP-1 expression in the absence of the $2 \mathrm{G}$ single nucleotide polymorphism is mediated by p38 and ERK1/2 mitogen-activated protein kinases in VMM5 melanoma cells," Journal of Cellular Biochemistry, vol. 86, no. 2, pp. 307-319, 2002.
[52] R. A. Cartlidge, G. R. Thomas, S. Cagnol et al., "Oncogenic $\mathrm{BRAF}^{\mathrm{V} 600 \mathrm{E}}$ inhibits BIM expression to promote melanoma cell survival," Pigment Cell and Melanoma Research, vol. 21, no. 5, pp. 534-544, 2008.

[53] K. M. Eisenmann, M. W. VanBrocklin, N. A. Staffend, S. M. Kitchen, and H. M. Koo, "Mitogen-activated protein kinase pathway-dependent tumor-specific survival signaling in melanoma cells through inactivation of the proapoptotic protein bad," Cancer Research, vol. 63, no. 23, pp. 8330-8337, 2003.

[54] J. T. Huntington, J. M. Shields, C. J. Der et al., "Overexpression of collagenase 1 (MMP-1) is mediated by the ERK pathway in invasive melanoma cells: role of $B R A F$ mutation and fibroblast growth factor signaling," Journal of Biological Chemistry, vol. 279, no. 32, pp. 33168-33176, 2004.

[55] M. Kono, I. S. Dunn, P. J. Durda et al., "Role of the mitogenactivated protein kinase signaling pathway in the regulation of human melanocytic antigen expression," Molecular Cancer Research, vol. 4, no. 10, pp. 779-792, 2006.

[56] S. M. Kumar, H. Yu, R. Edwards et al., "Mutant V600E BRAF increases hypoxia inducible factor- $1 \alpha$ expression in melanoma," Cancer Research, vol. 67, no. 7, pp. 3177-3184, 2007.

[57] A. Sharma, M. A. Tran, S. Liang et al., "Targeting mitogenactivated protein kinase/extracellular signal-regulated kinase kinase in the mutant (V600E) B-Raf signaling cascade effectively inhibits melanoma lung metastases," Cancer Research, vol. 66, no. 16, pp. 8200-8209, 2006.

[58] A. Sharma, N. R. Trivedi, M. A. Zimmerman, D. A. Tuveson, C. D. Smith, and G. P. Robertson, "Mutant ${ }^{\mathrm{V} 599 \mathrm{E}} \mathrm{B}-\mathrm{Raf}$ regulates growth and vascular development of malignant melanoma tumors," Cancer Research, vol. 65, no. 6, pp. 2412-2421, 2005.

[59] M. S. Soengas and S. W. Lowe, "Apoptosis and melanoma chemoresistance," Oncogene, vol. 22, no. 20, pp. 3138-3151, 2003.

[60] H. Sumimoto, F. Imabayashi, T. Iwata, and Y. Kawakami, “The BRAF-MAPK signaling pathway is essential for cancerimmune evasion in human melanoma cells," Journal of Experimental Medicine, vol. 203, no. 7, pp. 1651-1656, 2006.

[61] D. Woods, H. Cherwinski, E. Venetsanakos et al., "Induction of $\beta 3$-integrin gene expression by sustained activation of the Ras-regulated Raf-MEK-extracellular signal-regulated kinase signaling pathway," Molecular and Cellular Biology, vol. 21, no. 9, pp. 3192-3205, 2001.

[62] X. D. Zhang, J. M. Borrow, X. Y. Zhang, T. Nguyen, and P. Hersey, "Activation of ERK1/2 protects melanoma cells from TRAIL-induced apoptosis by inhibiting Smac/DIABLO release from mitochondria," Oncogene, vol. 22, no. 19, pp. 2869-2881, 2003.

[63] J. Newton-Bishop and N. Gruis, "Melanoma susceptibility genes," Melanoma Research, vol. 20, no. 3, pp. 161-162, 2010.

[64] D. T. Bishop, F. Demenais, M. M. Iles et al., "Genomewide association study identifies three loci associated with melanoma risk," Nature Genetics, vol. 41, no. 8, pp. 920-925, 2009.

[65] M. Falchi, V. Bataille, N. K. Hayward et al., "Genome-wide association study identifies variants at 9p21 and 22q13 associated with development of cutaneous nevi," Nature Genetics, vol. 41, no. 8, pp. 915-919, 2009. 
[66] I. Puzanov, K. L. Nathanson, P. B. Chapman et al., "PLX4032, a highly selective ${ }^{\mathrm{V} 600 \mathrm{E}}$ BRAF kinase inhibitor: clinical correlation of activity with pharmacokinetic and pharmacodynamic parameters in a phase I trial," Journal of Clinical Oncology, vol. 27 , no. 15 s, p. $9021,2009$.

[67] F. S. Hodi, P. Friedlander, C. L. Corless et al., "Major response to imatinib mesylate in KIT-mutated melanoma," Journal of Clinical Oncology, vol. 26, no. 12, pp. 2046-2051, 2008. 


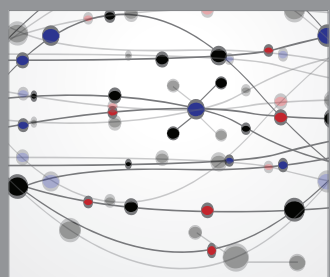

The Scientific World Journal
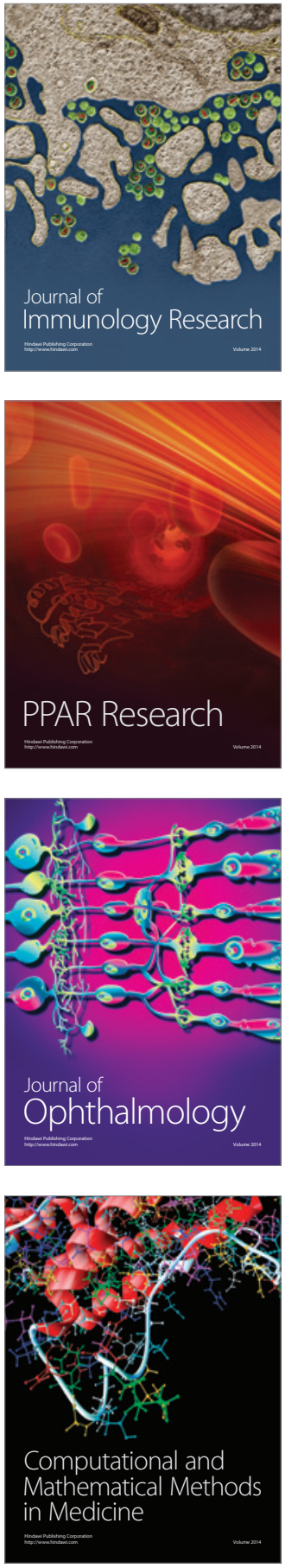

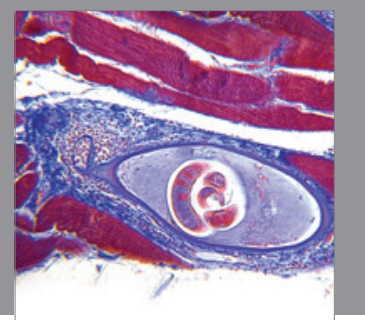

Gastroenterology

Research and Practice
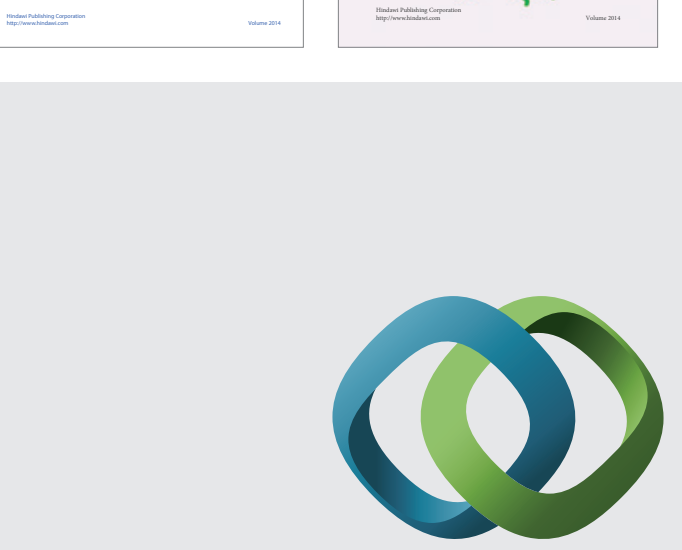

\section{Hindawi}

Submit your manuscripts at

http://www.hindawi.com
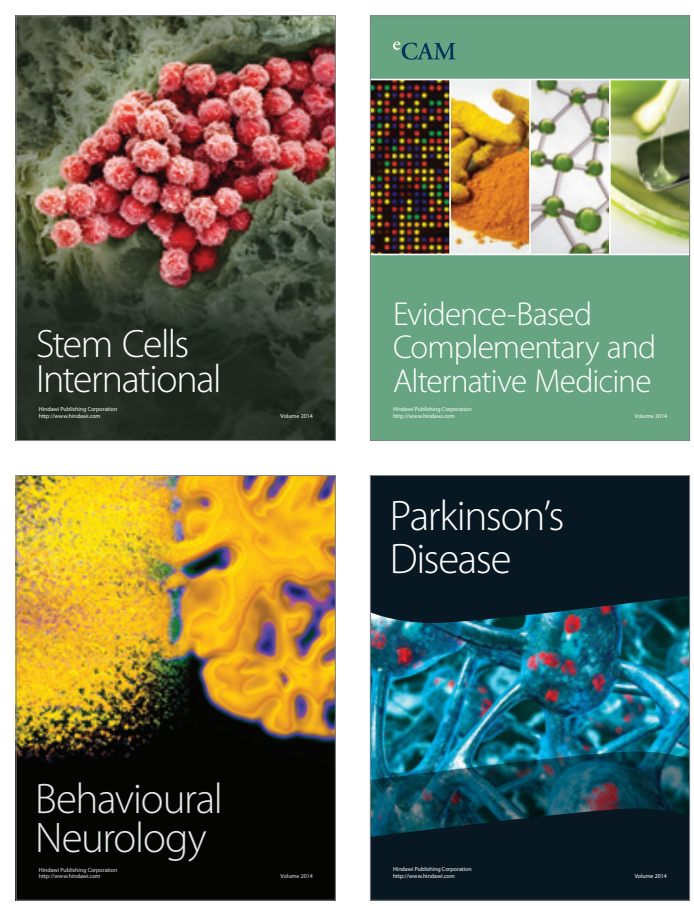

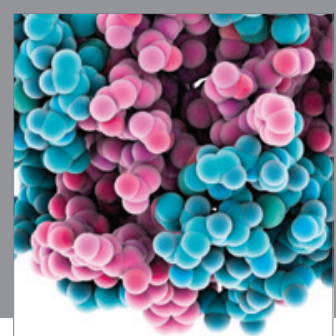

Journal of
Diabetes Research

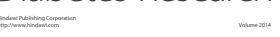

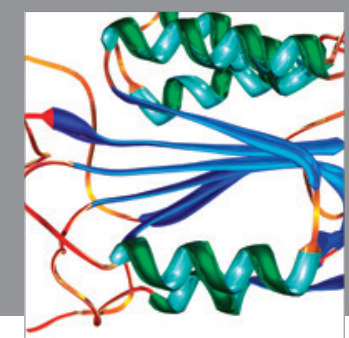

Disease Markers
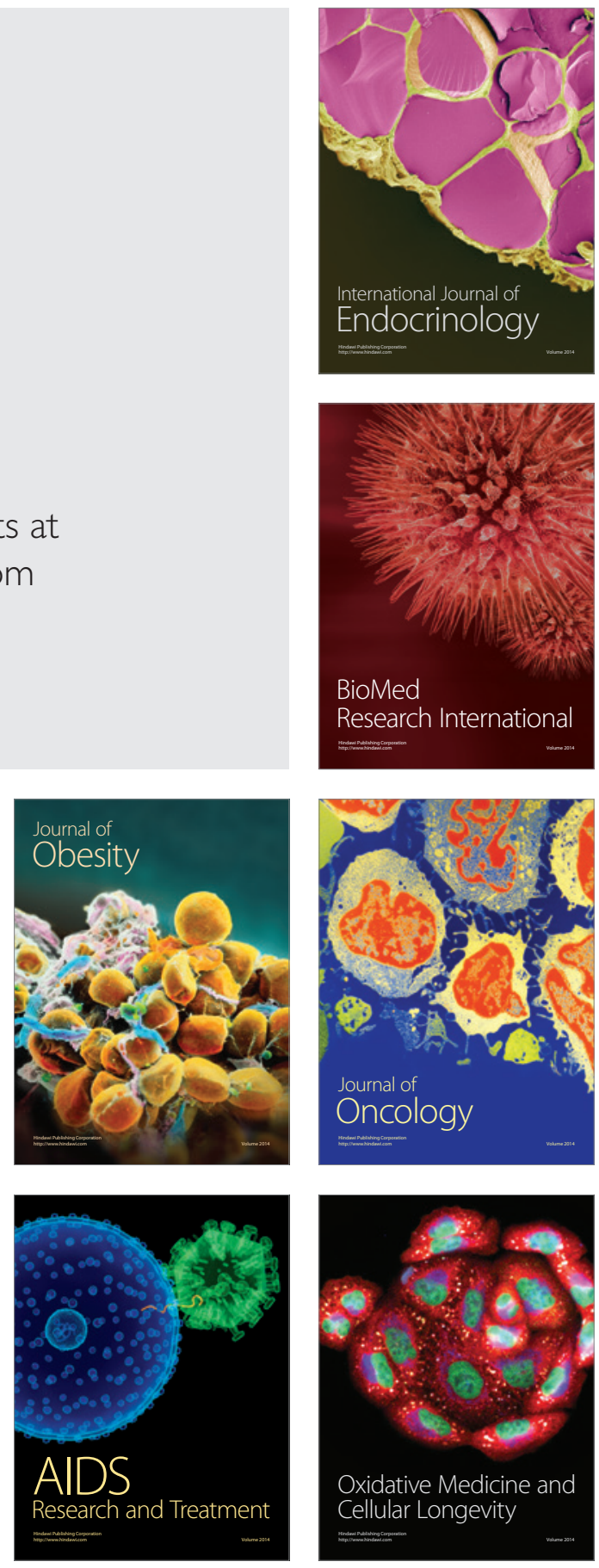